\title{
EXPERIENCIAS DEL DESASOSIEGO: SALUD MENTAL Y MALESTAR EN CHILE
}

Roberto Aceituno Morales, Gonzalo Miranda Hiriart

y Álvaro Jiménez Molina 


\section{ROBERTO ACEITUNO MORALES}

Psicólogo, Universidad de Chile, y Doctor en Psicopatología y Psicoanálisis, Universidad de Paris VII Diderot. Profesor asociado de la Facultad de Ciencias Sociales, Departamento de Psicología, y académico de la Facultad de Medicina, Departamento de Psiquiatría Sede Sur, Universidad de Chile. Responsable adjunto del proyecto Núcleo Bicentenario "Políticas del sujeto: malestar en la cultura, salud mental y vida cotidiana en Chile", Laboratorio Interdisciplinario de Prácticas Sociales y Subjetividad (LAPSOS), Universidad de Chile.Contacto: raceitun@uchile.cl

\section{GONZALO MIRANDA HIRIART}

Psicólogo, Magíster en Psicología Clínica (Pontificia Universidad Católica de Chile) y Doctor (C) en Salud Pública (Universidad de Chile). Profesor asociado de la Escuela de Psicología de la Universidad Católica Silva Henríquez y académico del Magíster en Psicología Clínica de Adultos, Universidad de Chile.

\section{ÁLVARO JIMÉNEZ MOLINA}

Licenciado en Psicología, Magíster (c) en Psicología Clínica de Adultos (Universidad de Chile). Investigador joven del Centro de Investigación Vulnerabilidades y Desastres Socionaturales (CIVDES), Universidad de Chile/Iniciativa Científica Milenio/ Ministerio de Economía, y miembro del Laboratorio Interdisciplinario de Prácticas Sociales y Subjetividad (LAPSOS), Universidad de Chile. 


\section{EXPERIENCIAS DEL DESASOSIEGO: SALUD MENTAL Y MALESTAR EN CHILE}

Un sentimiento generalizado de desasosiego recorre Chile.

Nuestro país muestra positivos indicadores económicos y sociales en los últimos treinta años: el ingreso per cápita ha pasado de 5 a 16 mil dólares, se ha masificado la educación, existe mayor acceso a la vivienda, el consumo no detiene su expansión y se ha consolidado un elevado índice de desarrollo humano. Y sin embargo, cada vez es más común escuchar hablar de un sentimiento difuso de "malestar".

La experiencia chilena de desasosiego ha venido acompañada por la instalación de un lenguaje del malestar que parece encontrar en la gramática de la "salud mental" una forma privilegiada de expresión: ya sea bajo la forma de indicadores epidemiológicos (ansiedad, depresión, suicidio, etc.), en la demanda creciente de atención en salud mental (psiquiátrica y/o psicoterapéutica) o en el aumento acelerado de licencias médicas por causas psiquiátricas.

Tales indicadores exigen una reflexión complementaria al abordaje médico-psicológico, a fin de instalar perspectivas pluridisciplinarias para el estudio antropológico y psicosocial del marco de las condiciones del malestar en Chile. Desde este punto de vista, la salud mental -tanto a nivel individual como colectivo- requiere de un análisis complejo que la articule a otros fenómenos de relevancia social. En efecto, la así llamada salud mental -o su reverso, el trastorno o padecimiento psíquico- supone experiencias subjetivas cuya expresión, pudiendo llegar a constituir sintomatología, se encuentra vinculada, en otro nivel de análisis, a las condiciones históricas de lo que Freud (1930) denominaba el "malestar en la cultura". En Chile, esta compleja trama de experiencias -a la vez individuales y colectivas- ha sido analizada, entre otros aspectos, desde una lógica donde la integración psicosocial de los individuos a un ordenamiento dado no opera sin un resto de malestar como "precio" subjetivo inherente al pacto social moderno.

En este sentido, diversos diagnósticos han identificado distintas fuentes para el malestar en Chile. Para algunos, el malestar sería un efecto de diferentes privaciones en las condiciones de vida (desigualdad) implicadas en el esfuerzo de integración social. Para otros, sería el efecto de una sociedad donde existe una democracia política cerrada sin una verdadera democratización social producto de la persistencia de "enclaves autoritarios" heredados de la dictadura militar. Asimismo, se ha planteado el malestar como resultado de prácticas neoliberales de administración del modelo económico y del trabajo, que promueven el retiro del Estado y la reducción de la protección social, multiplicando las desigualdades, debilitando la confianza frente a las instituciones políticas y subordinando las diferentes dimensiones de la vida social a la racionalidad (desregulada) del mercado. Por otro lado, el malestar se ha pensado como el efecto de una "inflación de expectativas", inevitablemente frustradas en el contexto de una sociedad de consumo que no responde a todas las demandas de satisfacción, o como el efecto de las "paradojas de la modernización" (PNUD, 1998), es decir, de un desajuste entre un 
proceso de modernización impuesto -acelerado y naturalizado- que produce nuevas oportunidades, pero al mismo tiempo genera inseguridades y miedos. Recientemente ha aparecido la idea del malestar como "indignación" asociada al abandono de valores materiales ligados a la seguridad económica y la emergencia de valores "post-materiales" vinculados a las demandas de mayor participación y autonomía: la expansión de una "nueva clase media" habría desplazado la "cuestión social" desde la reivindicación de la igualdad hacia la búsqueda del "bienestar". Finalmente, para el caso específico de los jóvenes, el malestar sería el resultado de diferentes dificultades en el acceso a la educación superior, al mercado del trabajo y a los espacios de participación de la sociedad, a causa de una crisis de representación de los sistemas políticos tradicionales.

En síntesis, se han desarrollado explicaciones estructurales (desigualdad económica), políticas (democracia deficiente) y culturales (nuevos valores) del malestar, las cuales subrayan diferentes dimensiones de la sociedad. Sin embargo, no han faltado investigadores que sostienen que el "discurso del malestar" en Chile no es más que una reacción ideológicamente neo-conservadora al proceso de modernización, la cual parece tener nostalgia por una suerte de comunidad perdida (Brunner, 1998).

Desde el punto de vista asumido en este artículo, es preciso sin embargo introducir otros criterios para el análisis del problema, en la medida que tanto el malestar colectivo como sus expresiones "psíquicas" - eventualmente "psicopatológicas"- se basarían hoy en día en condiciones diferentes a la simple oposición entre bienestar y malestar o entre expectativas y acceso a niveles de desarrollo (material o humano) diferenciados como algo inherente a los procesos de modernización. Tales condiciones conciernen a características del lazo social contemporáneo que inciden en una individualización creciente del malestar. Y ello en un doble sentido. En primer lugar, por el modo en que es experimentado el malestar por los sujetos en su ámbito "privado" y, en segundo lugar, por la manera a través de la cual los discursos sociales actuales construyen ideales de bienestar y de adaptación, donde el individuo es el responsable ilusorio de un desarrollo provisto "clásicamente" por un marco de sociabilidad donde el progreso individual es inseparable de condiciones sociales, económicas, políticas y culturales. En este sentido, la individualización del malestar -y de la salud mental- es correlativa a contradicciones y conflictos característicos de la vida social contemporánea, lo cual se traduce en la experiencia de desconfianza, de miedo (o angustia) y de debilitamiento de un sentido propiamente humano de la vida individual y colectiva.

\section{PREVALENCIA DE TRASTORNOS MENTALES EN CHILE: ¿EL PRECIO DEL ÉXITO?}

Sin duda, la centralidad de la salud mental en los discursos del malestar está asociada a un cambio en el perfil epidemiológico de las sociedades. Allí donde a comienzos del siglo XX las enfermedades típicas eran las infecciosas, en las sociedades contemporáneas son cada vez más las enfermedades crónicas y las enfermedades mentales.

Todo indica que en Chile nos encontramos actualmente en un proceso de transición epidemiológica asociado a un cambio en la estructura poblacional propia de los países 
en desarrollo. Una de las hipótesis básicas de la epidemiología social es que la absorción de las tensiones que aparecen en los procesos de cambio que implica el crecimiento económico, están asociadas a la aparición de síntomas psiquiátricos y psicosomáticos (Almeida-Filho, 1998).

¿Qué nos dicen los datos? Si nos detenemos en los estudios epidemiológicos, observamos que en Chile una de cada tres personas sufre problemas de salud mental en algún momento de su vida, mientras que una de cada cinco ha tenido un desorden en los últimos seis meses, principalmente trastornos ansiosos y depresivos (Vicente, 2002). Sin duda esto constituye un problema para el desarrollo del país, puesto que los costos asociados a los trastornos mentales oscilan entre un 3 a 4\% del PIB, según estimaciones para los países desarrollados (OMS, 2001). En Chile, el costo más significativo es el que representan las pérdidas de productividad por los años de vida saludables perdidos, donde los trastornos neuropsiquiátricos contribuyen con el 31\%, siendo uno de los índices más altos en el mundo (Vicente et al., 2007).

Si bien la mayoría de los indicadores de prevalencia de patología psiquiátrica en Chile se encuentran en la media de los países de América Latina, nuestro país duplica la tasa regional de prevalencia de distimia ( $8 \%$ v/s 3,5\% en América Latina) y abuso o dependencia de drogas ilícitas (3,5\% v/s 1,6\% en América Latina) durante la vida (Kohn et al., 2005). En Chile, la prevalencia de síntomas depresivos en la población es de 17,2\%. Entre la población adulta, un 21,67\% reporta haber recibido diagnóstico médico de depresión alguna vez en la vida, lo que tiene una tendencia a ser mayor entre las mujeres y en el grupo de menor nivel educacional (MINSAL, 2011b). No resulta sorprendente entonces que estadísticas de la OMS (2001) señalen que Santiago de Chile encabeza las capitales con mayor número de trastornos ansiosos y depresivos en el mundo. Esto se ha traducido en un aumento explosivo del orden de 470,2\% en el consumo de antidepresivos entre 1992 y 2004 (Jirón et al. 2008). Por cierto, ello podría deberse a distintas causas: desde mayor oferta farmacológica o diferente manejo de los trastornos anímicos.

Un aspecto específico del problema se expresa, según estadísticas de la OCDE (2011), en el hecho de que la tasa de suicidio en Chile ha aumentado en un 55\% entre 1995-2009. Chile es el país de la OCDE donde más ha aumentado el suicidio, después de Corea del Sur. En Chile mueren más de 1.500 personas al año por suicidio, y la mortalidad por heridas auto-provocadas intencionalmente ha aumentado entre 1990 y 2005 desde 5,7 a 9,3 por cada 100.000 personas (Rojas, 2008). ¿Qué nos dice este hecho acerca de nuestra sociedad? El perfil del suicida chileno ha sido definido como una persona que ha acumulado una carga de hostilidad que puede originarse a partir de las dificultades en el proceso de integración en la sociedad, lo cual permite entender su conducta como un hecho violento dirigido en contra de sí mismo (Duarte, 2007). De hecho, en Chile aumenta la conducta suicida al mismo tiempo que aumenta la conducta homicida. Esto puede ser leído como el reflejo de la acumulación de un malestar que lleva a una mayor violencia. Algunos concluyen que esto sería síntoma de exclusión social. Análisis estadísticos arrojan que existe una alta y significativa correlación entre el número de suicidios y el aumento del PIB en el período 1981-2003, un resultado que permite 
inferir que el suicidio se encuentra estrechamente asociado a un proceso de desarrollo económico atravesado por profundas desigualdades (Moyano y Barría, 2006). En este sentido, un factor clave para entender las variaciones en las tasas de suicidio parece ser el proceso de crecimiento económico acompañado de modificaciones importantes en el mercado del trabajo durante los últimos veinte años. De hecho, las cifras más altas de prevalencia de trastornos mentales se encuentran en ciudades que han tenido un acelerado desarrollo económico.

La población más vulnerable a sufrir problemas de salud mental pareciera ubicarse en el segmento de los adultos en plena edad productiva (44-54 años). En este sentido, la mayor prevalencia de enfermedades mentales y del comportamiento ha provocado parte importante del aumento en la cantidad de licencias médicas en Chile. Los problemas psicológicos se convirtieron a partir del año 2008 en la primera causa de incapacidad transitoria entre los beneficiarios del sistema público de salud. En las ISAPRE, los trastornos mentales representaban el 2005 un 15,3\% del total de licencias, incrementando el 2008 a 20,4\%, convirtiéndose así en el grupo patológico con mayor peso relativo (Miranda et al., 2012). De este modo, uno de cada tres días no trabajados por razones de salud se debe a problemas mentales. Sin embargo, durante los últimos años se observa un aumento importante de patologías mentales en niños, jóvenes y adultos jóvenes. De hecho, las tasas de suicidio históricamente más elevadas entre las personas mayores, actualmente tienen la tendencia a invertirse hacia los grupos más jóvenes (Rojas, 2008). Asimismo, los comportamientos de automutilación (heridas auto-provocadas, cortes, etc.) son cada vez más frecuentes entre los adolescentes y representarán en el futuro una parte importante de la demanda de atención en psiquiatría. Si nos detenemos en este segmento de la población, encontramos que los resultados son preocupantes: según un reciente estudio nacional de calidad de vida y bienestar de los jóvenes, los puntajes más bajos se encuentran en la dimensión bienestar psicológico, estado de ánimo y emociones, especialmente en mujeres (Molina et al., 2012), mientras que según un estudio nacional de prevalencia psiquiátrica infanto-juvenil, un 22\% de niños y adolescentes chilenos presenta algún tipo de trastorno psiquiátrico (de la Barra et al., 2012).

En paralelo, se produce una mayor demanda de atención psicológica y psiquiátrica: una de cada tres consultas en todo el servicio público de Santiago está dada por trastornos ansioso-depresivos, persistiendo aún una amplia brecha (diferencia entre las tasas de prevalencia $\mathrm{v} / \mathrm{s}$ las personas tratadas) en el tratamiento de los trastornos mentales (Kohn et al., 2005).

La prevalencia de problemas en salud mental es un problema para la sociedad chilena en su conjunto, y para las políticas públicas en particular. Más aun cuando existe una relación inversa entre estrato socioeconómico y la prevalencia de muchos trastornos psiquiátricos, lo cual podría verse reflejado también en el acceso a atención especializada. De hecho, en Chile existen importantes brechas en las percepciones de calidad de vida según grupos socioeconómicos: a medida que aumenta el bienestar socioeconómico, aumenta la probabilidad de tener un mejor estado de salud percibido (MINSAL, 2006). 


\section{HIPÓTESIS PARA UN ANÁLISIS DE LA RELACIÓN ENTRE SALUD MENTAL Y MALESTAR EN CHILE}

Los problemas de salud mental en Chile no solo requieren de estudios e intervenciones que se sitúan en el ámbito clínico, sino que también deben ser analizados en función de las condiciones relativamente específicas a la subjetividad y a las relaciones sociales contemporáneas.

Como ya se ha dicho, es posible que estas condiciones socio-culturales conciernan no solo a la insatisfacción respecto a las promesas incumplidas (o cumplidas solo parcialmente) de desarrollo ofrecidas por los modelos de integración prevalecientes en las sociedades "de consumo" o "de mercado", sino también a una individualización de la gestión de desarrollo clásicamente organizada en torno a las mínimas garantías dadas por el Estado, las políticas públicas y una lógica de derechos. Dicha individualización sitúa "al interior" de los sujetos (por lo tanto en su espacio "psíquico") un ideal que se vuelve imperativo y persecutorio, como exigencia de emprendimiento, de bienestar y, sobre todo, de rendimiento (o competencia), transformando al sujeto en objeto de imperativos producidos socialmente, pero que se experimentan, por así decirlo, "al interior" del sujeto mismo. Los discursos -es decir, ideologías y prácticas- de las economías neoliberales desplazan la lógica del malestar desde el ámbito socio-político al de una psicopolítica del individuo, enfrentado ahora menos a un orden social dado que a sus propias expectativas (y exigencias) de consumo y, también, al desencuentro con el otro, que se vuelve el vecino-adversario de su aparente bienestar. La experiencia clínica más cotidiana demuestra que las demandas de salud (mental, en este caso) son el territorio donde la subjetividad, vaciada de su sentido a la vez individual y comunitario, se dirige a interlocutores institucionales diversos (el "Otro" social) para encontrar ahí no solo la respuesta al contenido de tales demandas sino un reconocimiento -improbable- de su propia legitimidad.

Los "síntomas" de deterioro de la salud mental prevalentes en nuestra sociedad y en nuestra época pueden ser considerados a modo de hipótesis como la expresión "psicopatológica" de un estado de malestar que va más allá de sus componentes puramente individuales. O, más bien, donde el espacio "psíquico" es a la vez la traducción aparentemente "privada" de demandas que caracterizan la relación al Otro social en la cotidianeidad de la experiencia social. En este sentido, es preciso reconocer que los "síntomas" no son necesariamente "signos" de enfermedades o de trastornos que la clínica médica o psicológica se encargaría de revelar y de tratar terapéuticamente, sino que dan cuenta de una dinámica -a la vez individual y colectiva- por la cual se constituyen como mensajes y demandas dirigidas al Otro social en sus distintos niveles -desde el semejante en las relaciones intersubjetivas al Otro social representado institucionalmente- organizadas en torno a una lógica que denominaremos de reconocimiento. Dicho reconocimiento, vehiculizado por el malestar y el sufrimiento psíquico, no concierne únicamente al "contenido" de las demandas de salud (alivio sintomático, resolución de conflictos, estados de satisfacción y de bienestar), sino que, sobre todo, apunta al reconocimiento del sujeto ahí implicado y a su inscripción "simbólica" a nivel de la sociedad y de la cultura. Cuando la 
respuesta (o no respuesta) a estas demandas se sitúa únicamente en la dimensión de la satisfacción (o, más críticamente, en el desconocimiento) de las necesidades de desarrollo (o de las demandas de reconocimiento subjetivo), la salud mental resulta un problema privado encerrado en el emprendimiento individual o en una lógica subsidiaria provista por un Estado sordo y ciego a la subjetividad ahí implicada.

Un segundo elemento a considerar para el análisis del problema se refiere específicamente al "miedo" como rasgo que acompaña la experiencia subjetiva de malestar y a los vínculos intersubjetivos o, más ampliamente, a la experiencia del lazo social contemporáneo.

Los temores básicos descritos por el Informe de Desarrollo Humano de 1998 del Programa de Naciones Unidas para el Desarrollo (PNUD): miedo al otro, a la exclusión social o al sinsentido producto de la contradicción entre valores y condiciones materiales, incluyeron la noción de "seguridad humana" como nuevo elemento de análisis. El miedo al otro se hacía visible en los estudios hace ya 15 años a través de un miedo exacerbado a la delincuencia -que contrasta con las cifras efectivas-, lo que parecía estar relevando otras inseguridades; aquellas provocadas por el debilitamiento del vínculo social, el sentimiento de comunidad y, finalmente, de la noción misma de orden (PNUD, 1998). Pero además, agrega el informe, existiría un malestar social creciente, asociado, por una parte, a la falta de confianza en los sistemas (entre ellos los sistemas de salud y previsional), motivada por la percepción de que dichos sistemas funcionan mal y distribuyen de manera desigual oportunidades y riesgos. Es decir, no se confía en estos porque no cumplen a cabalidad sus respectivas funciones. Por otra parte, dicho malestar estaría asociado a una vinculación demasiado contractualista e instrumental entre las personas y dichos sistemas. Un enfoque percibido como excesivamente "economicista" estaría descuidando otras necesidades de los individuos, como los lazos de confianza, las relaciones de gratuidad y solidaridad, el respeto, la pertenencia y, en general, toda la trama propia de la sociabilidad cotidiana. Se aprecia la sensación de que las personas pueden ser instrumentalizadas en función de la mayor competitividad y productividad de los sistemas (PNUD, 1998).

Hoy, podemos decir que el miedo al otro no ha cedido y que la confianza en los sistemas se ha continuado deteriorando. Más aun, que ya no es solo la sociabilidad la que se ha debilitado, sino el pacto social mismo; la autoridad ya no parece ser garante del bien común y la democracia ha sufrido un desprestigio importante. Si hace 15 años se percibía que los sistemas funcionaban mal, y que las relaciones entre los sujetos y las instituciones eran instrumentales y excesivamente contractualistas, hoy la percepción es más bien que las organizaciones esconden oscuras intenciones, y que el temor ya no es solo a lo otro, a lo diferente, sino que se ha extendido a aquello que parecía familiar y conocido.

La exacerbación de las demandas individuales, la sensación de estar moviéndose en una trama de lazos deshonestos, más la falta de reconocimiento en las instituciones políticas, acercan al sujeto a la paranoia, el cinismo y a la práctica de una violencia sin estrategia. Sin duda, por alguna parte eso se tiene que traducir en formas de sufrimiento psíquico y llegar finalmente a los servicios y profesionales que acogen problemas de salud mental. 


\section{MODERNIZACIÓN E INDIVIDUALIZACIÓN DEL MALESTAR}

En el proceso de modernización las sociedades occidentales se volvieron más individualistas. Modernización e individualismo parecen estar al centro de los ejes de lectura del malestar social y los nuevos desafíos en salud mental. En este debate las posiciones son disímiles. Hay quienes sostienen que las sociedades más individualizadas tienden a ser más felices (Veenhoven, 1999), y tal como lo sostiene el modelo del "desarrollo humano", los factores que favorecen la libertad de elección, como el desarrollo económico y la democratización, conducen a niveles crecientes de bienestar subjetivo (Inglehart et al., 2008). Sin embargo, la posición dominante en Chile parece ser entender el malestar como una expresión típica de la modernidad producto de la escisión entre modernización y subjetividad como dos procesos autónomos (PNUD, 1998; Lechner, 2007). En este sentido, el proceso de modernización implicaría una crisis de la "cohesión social", en la medida en que traen consigo un cuestionamiento de los vínculos y creencias básicas de tipo comunitario y en torno a las expectativas de desarrollo.

Los diagnósticos en esta línea son diversos: las formas de trabajo propias del capitalismo tardío requieren de un nivel de flexibilidad y fluidez que genera incertidumbre e inestabilidad, desplazándose a distintas esferas de la vida, impactando así sobre un carácter vulnerable y predispuesto a la depresión (Sennet, 2002); producto de que los individuos deben ofrecer "soluciones biográficas a contradicciones sistémicas", en las sociedades postindustriales los problemas sociales se transformarían en disposiciones psíquicas: insuficiencia personal, sentimientos de culpa, miedos (Beck, 1986); el proceso de individualización radicalizado que supone la modernidad tardía aparecería como el trasfondo de desequilibrios subjetivos asociados al declive de las fuerzas interiores del individuo y a la pobreza psíquica del sujeto actual, lo cual se reflejaría en comportamientos individuales atrapados en la lógica del exceso propia de la modernidad tardía: frenesí consumista, obesidad, bulimias y anorexias, compulsiones, adicciones (Lipovetsky, 2006). El proceso de desinstitucionalización, desprotección, desregulación, privatización y responsabilización que implica la instalación del ideal de "autonomía" supone un abandono progresivo de la sociedad por el Estado, lo cual dejaría a los individuos frente a sus debilidades. En estos términos, los sufrimientos sociales serían un producto del cambio de las relaciones entre sociedad y Estado: allí donde el liberalismo clásico habría apostado a una complementariedad espontánea entre modernización y subjetividad, el modelo socialdemócrata pensó en la complementariedad por medio del Estado. En síntesis, se postula que el proceso progresivo de "individualización", al mismo tiempo que aumenta el ámbito de la autonomía individual, disminuiría la protección que brindaban las convenciones y normas sociales, produciendo riesgos y sentimientos de angustia.

Sin embargo, este tipo de diagnósticos -que gozan de relativa aceptación- presentan una serie de dificultades. Por un lado, confunden "individualismo" y "subjetividad", y en esa especie de "subjetividad líquida" que describen existiría una vulnerabilidad que parece depender de la declinación de los antiguos sistemas de estructuración de los sujetos. En este sentido, gran parte de los diagnósticos del malestar tienden a 
sostener un discurso de la degradación social: el malestar se resumiría en la doble idea de que el lazo social se debilita y que en contraparte el individuo es sobrecargado de responsabilidades y pruebas que no conocía antes. Esto los transforma en diagnósticos inherentemente conservadores. Por otro lado, las coordenadas de la individualización según la lógica de la (segunda/post/hiper) modernidad son altamente homogeneizadoras de las singularidades de las sociedades contemporáneas, particularmente de las latinoamericanas.

La sociedad es percibida por los chilenos como una fuente permanente de inseguridad. Pero el individualismo chileno nada tiene que ver con el europeo, porque los soportes necesarios para la individuación no se encuentran en las instituciones tradicionales. En Europa el proceso modernizador fue paulatino, lo que permitió una adecuación con los cuadros normativos, y ha producido un individuo que se sabe sostenido por un entramado social y una gobernabilidad que produce a la ciudadanía como actor, mientras que en Chile el individuo ha debido constituirse como un "hiper-actor" que debe sostener tanto su sobrevivencia material como la producción de sentido (Araujo et al., 2012). Si bien, la sociedad chilena es una de las más institucionalizadas de América Latina, ni la sociedad ni la producción de sus individuos pueden interpretarse desde una prescripción institucional central (trabajo, empleo, escuela, familia, Estado, etc.) que los "interpela" bajo un mandato normativo de "autonomía" en tanto sujetos. En Chile se trata más bien de un individuo que se produce por fuera de esas instituciones tradicionales. Si el "self" y la oportunidad en Estados Unidos, o la "institución" y la protección en Francia muestran dos polos del individualismo y sus formas de malestar (Ehrenberg, 2010), esta distinción es más compleja en los países de América Latina.

¿Cuál sería entonces la especificidad de Chile? Nuestro país fue el primer laboratorio mundial de un proyecto político, económico y social que hoy conocemos como "neoliberalismo". Nuestra vida social está atravesada por un conjunto de transformaciones ligadas al despliegue de dicho proyecto. En este sentido, interrogarse por el individuo estructuralmente producido en Chile implica preguntar por las dos grandes revoluciones del proceso modernizador, es decir, por la constante tensión entre una "revolución neoliberal incompleta" y una "revolución democratizadora inacabada". Y sin embargo no existe algo así como un "individuo neoliberal" en Chile (Araujo et al., 2012).

Los chilenos se desenvuelven dentro de un contexto (hiper)individualizado y mercantilizado que genera cierto hálito de anomia y alienación, y que exige de los propios individuos la producción del sentido necesario para un vida social incierta (Ruiz et al., 2011). De hecho, en el momento de dar cuenta de su "bienestar subjetivo", los chilenos atribuyen gran importancia a las características personales. Ello no solo habla del "neonarcisismo" de hoy, sino que muestra que para los chilenos la producción del malestar ya no parece residir tanto en la estructura social, sino más bien en las competencias individuales. Asimismo, los mayores obstáculos para lograr la felicidad se identifican en el registro de la relación al otro, mientras que la relación con las instituciones está marcada ya sea por una sensación de "desprotección" o de "abuso". En este contexto, los individuos se sienten protegidos solo al interior del principal ámbito institucional de la 
sociedad chilena: la familia, espacio que no deja de aparecer asociado en el discurso de los chilenos a las experiencias de bienestar/malestar.

La presencia de la lógica neoliberal y la desestabilización que produce a nivel de la experiencia de los individuos (flexibilidad laboral, generalización de la competencia, endeudamiento, etc.) se refleja en un doble desasosiego. Por un lado, en una "inconsistencia posicional" generalizada: nadie se siente seguro en su lugar debido a una percepción de que los emplazamientos, las trayectorias y la integración social son inestables (por ejemplo, es el caso del trabajo temporal, informal o en la subcontratación, etc.). En ese contexto solo queda arreglárselas mediante el esfuerzo personal. Por otro lado, el desasosiego se expresa bajo la forma de un "desequilibrio temporal", un sentimiento de pérdida de control de las capacidades de manejo del tiempo debido a una experiencia del trabajo en el que -literalmente- se consume la vida (Araujo et al., 2012).

Las formas de procesamiento o encausamiento del malestar es uno de los problemas centrales al momento de intentar comprender la forma que asume el malestar en una sociedad. En Chile, estatismo, despolitización y malestar se han movido en evoluciones semejantes en los últimos 20 años (Mayol y Azócar, 2011). Si bien el "apoliticismo" o la "despolitización" han sido elementos constitutivos del Chile de las últimas décadas, las movilizaciones estudiantiles muestran un hecho que debería parecer evidente: la forma privilegiada de elaboración del malestar debiera ser su politización. Sin embargo, el encausamiento por la vía de una comunidad política no parece ser la forma principal de elaboración del malestar en Chile. Tal vez por ello existe una tendencia cada vez más generalizada a redefinir múltiples problemas utilizando la gramática de la "vulnerabilidad individual". Dicho de otro modo, el auge de un lenguaje que expresa los conflictos sociales en términos de dificultades personales da cuenta de cierta "crisis de las retóricas políticas" para expresar la queja colectiva (Martuccelli, 2007).

Así como en el nacimiento de la sociedad industrial la confrontación política se expresaba en términos morales ("la cuestión social"), actualmente los conflictos y malestares sociales tienden a expresarse en términos psicológicos. De ahí precisamente la centralidad que adquiere la referencia a la "salud mental". Sin embargo, los diagnósticos tradicionales en la línea de la "segunda modernidad" impiden comprender los cambios normativos en la sociedad chilena, y dificultan la descripción de las transformaciones en las relaciones entre subjetividad, salud mental y lazo social. En la nueva dinámica que se establece entre lo psicológico y lo sociológico vía el malestar, las analíticas disponibles se revelan incapaces de establecer una demarcación nítida entre sufrimiento social y salud mental. Por lo tanto, la teoría social requiere de nuevos esquemas analíticos de interpretación del malestar que permitan releer el eje modernización/individualismo. Y en ello puede jugar un papel relevante introducir de una manera distinta una discusión en torno a la salud mental.

Ahora bien, el hecho de que el sufrimiento psíquico y la salud mental parezcan ser uno de los principales puntos de referencia de la individualización contemporánea, y que los problemas parezcan "más personales", no es sinónimo de que sean menos sociales, menos políticos o institucionales, sino que lo son de otra manera. Después de todo, el "malestar" es también una cierta representación colectiva que la sociedad genera 
acerca de sí misma. Esto se refleja, por ejemplo, en el hecho de que en la sociedad francesa el malestar se exprese bajo la forma de "sufrimiento", mientras que en Chile asume la forma de "miedo" (Arteaga et al., 2012).

\section{LAS RESPUESTAS AL MALESTAR}

En el curso de los últimos veinte años un conjunto de sufrimientos psíquicos aparecen con fuerza en el espacio público: depresión, crisis de pánico, ansiedad generalizada, sufrimientos en el trabajo, consumo de drogas, impulsiones suicidas, etc. Como un proceso paralelo, hoy en día el espacio público se ve invadido por un conjunto de ofertas terapéuticas de todo tipo (que van desde la psicoterapia al yoga o los libros de autocuidado). Y lo que sería cómico si no fuera tan trágico, es que en este nuevo mercado la felicidad misma se ha transformado en un objeto de consumo. De este modo, pareciera existir un estrecho vínculo entre el imperativo del bienestar y el aumento del malestar. Ya lo decía Freud (1930): cuanto más nos sometemos a la presión de los ideales, más malestar experimentamos.

Si la noción de salud mental ocupa un lugar cada vez más central en la vida social, es porque da cabida a un conjunto amplio de problemas que no conciernen solo a la salud, sino a la sociabilidad misma (en la escuela, en el trabajo, etc.).

Si la hipótesis de que existe una vinculación estrecha entre el malestar social y algunos trastornos mentales es correcta, se plantea entonces el desafío de mejorar los índices de salud mental en Chile a través de una acertada gestión de lo social. O dicho de otro modo, para abordar el deterioro de la salud mental de los chilenos no es suficiente aumentar la cobertura de atención psicológica y psiquiátrica en los servicios de salud si no se interviene sobre el contexto: económico, social y cultural.

Por lo demás, esta no es una idea nueva. La tradición de la medicina social promueve desde el siglo XIX un enfoque donde la estructura social pasa a ser parte central en la explicación de la enfermedad; por lo tanto, la acción política entra en el campo de la medicina, y las reformas sociales dentro del interés y de las obligaciones de los profesionales de la salud. Más recientemente, la Comisión de Determinantes Sociales de la Salud, creada por la OMS (2008), llegó a la conclusión de que es urgente actuar directamente sobre la desigualdad socioeconómica como vía para mejorar la salud en general. El problema es a qué nivel y cómo intervenir en lo social, pues es algo tan vasto como impreciso. Las explicaciones sobre el origen del malestar son variadas, y los caminos posibles para hacer algo son tantos como inciertos.

En tiempos donde las propuestas propiamente políticas pierden fuerza, y descontando los intentos por avanzar hacia una sociedad más equitativa, dos son los enfoques que -proviniendo de la academia- intentan proveer de un marco comprensivo para las políticas públicas: el de capital social (Coleman, 1990), que proviene de la sociología, y el de la felicidad (Layard, 2005), que proviene de la psicología y la economía. Hasta ahora, ambos enfoques se han convertido en agregados de políticas, y sobre todo de mediciones, enmarcadas en un paradigma modernizador. Por lo tanto, se han transformado 
más bien en un problema de estadísticas y de indicadores matemáticos, que dejan pendiente los debates ideológicos y éticos que están a la base, incluyendo el tratamiento poblacional que se hace del capital social y de la felicidad, que tiende a indiferenciar a los sujetos y estandarizar las prácticas.

La salud mental sigue relegada al ámbito sanitario. Cuestión que no es extraña en Chile, pues el sistema de salud -uno de los más antiguos del mundo- cuenta con una alta legitimidad y es referente en todo el país para el tratamiento de una gran diversidad de dolencias y problemas. De este modo, es imposible saber en qué medida se están medicalizando formas variadas de sufrimiento, en especial, desde que la depresión cuenta con cobertura GES. Ahora bien, como sabemos, la gestión se ha ido imponiendo a la clínica en los sistemas de salud. Obligados a dar cuenta de su productividad, hoy en día la atención sanitaria se ve limitada en términos de la evidencia dominante y por el criterio de costo-efectividad. Eso se traduce en una taylorización de la práctica asistencial, cada vez más sorda a la narrativa de quienes piden ayuda, y para la cual las diferencias individuales son una molestia. Si la hipótesis es que el marco social simbólico es cada vez más deficitario como para poder construir experiencia y subjetividad, esta manera de hacer medicina solo agravaría el problema del malestar. Si de verdad estamos ante una nueva cuestión social, también debería haber una nueva manera de encarar la acción asistencial del Estado.

La alianza entre una biomedicina cada vez más tecnificada -y menos atenta a la relación entre paciente y tratante- con la economía de la salud y la medicina basada en evidencia, lo que está haciendo es profundizar el desencuentro entre la demanda de quien solicita ayuda y la oferta de los servicios de salud. En la Atención Primaria, donde se espera que se "resuelvan" la mayor cantidad de casos, se vive a diario esta experiencia; los profesionales consideran injustificadas una alta proporción de las consultas, que trascienden las competencias del equipo, y -lo que es una novedad- que afectan la productividad de los establecimientos. Ante eso, los casos son etiquetados como "trastornos de la personalidad" o "psicosociales".

Lo psicosocial se ha convertido así en un recurso ante la dificultad de abordar comprensivamente ciertas formas de sufrimiento que no serían legítimamente enfermedades o condiciones de salud, que termina banalizando el malestar de muchas personas que se acercan a los servicios de salud (Saffie, 2011). Al pasar lo psicosocial por el cedazo de la evidencia primero, y de las políticas luego, se simplifica hasta el absurdo, y se reduce la clínica más trabajosa posible, la del sufrimiento psíquico, a prácticas de segunda categoría, que no se condicen con la complejidad de su origen. Es posible ofrecer respuestas individuales al malestar social; es más, es una necesidad y una exigencia de los tiempos, pero no puede ser agravando la condición del sujeto que sufre.

Abordar entonces el problema de la salud mental en Chile requiere someter a análisis no solo las formas -extremas o difusas- del malestar, sino ligar dicha experiencia a las respuestas institucionales provistas por el Estado, es decir, a nivel de las políticas públicas. En este nivel de análisis, dichas respuestas forman parte del problema mismo que buscarían resolver, en la medida que las demandas expresadas por los "trastornos" mentales encontrarían un espacio de reconocimiento político acorde a la complejidad 
del problema, o bien un desconocimiento que redoblaría el malestar mismo. Asimismo, la crisis de confianza que hemos mencionado como uno de los elementos antropológicos del malestar (y de la salud mental) en Chile hoy, como el desasosiego frente a un estado de cosas que banaliza el sufrimiento mediante una lógica economicista y pseudo-productiva, se traduciría en la esfera de un diálogo improbable entre malestar y modernización.

\section{A MODO DE CONCLUSIÓN}

La salud mental parece estar en el horizonte del individualismo contemporáneo y la expansión de su discurso ha hecho que la subjetividad individual devenga progresivamente en una cuestión colectiva. En efecto, la salud mental en Chile constituye un problema de relevancia a la vez subjetiva y sociocultural. Ello se expresa tanto a nivel de los indicadores epidemiológicos mencionados, como, por otra parte, en su estrecha relación con las condiciones económicas, políticas y culturales prevalecientes en una sociedad donde la integración (y exclusión) social produce crecientes niveles de malestar. El incremento de trastornos "psicológicos" operaría entonces menos como signo de una modernización acelerada -cuya dinámica psicosocial admitiría mayores niveles de expectativas de desarrollo incompatibles con la satisfacción inmediata de las demandas que tal modernización crearía-, que como la expresión subjetiva de conflictos y contradicciones que la lógica "moderna" clásica no permite analizar del todo, en función de nuevas formas del "malestar en la cultura".

En esta perspectiva, la complejidad del problema, como de sus posibles abordajes políticos, requiere considerar al menos dos criterios de análisis. El primero apunta a considerar que los trastornos psíquicos no son el precio inevitable de una modernización que, a través de un incremento de las expectativas de desarrollo, conduciría a un desasosiego que el tiempo podría calmar cuando el desarrollo social -promesa política frecuentemente aludida- se cumpliera efectivamente. En cambio, expresan una crisis en la credibilidad de tales promesas, un efecto privado de las políticas públicas vaciadas de reconocimiento a las demandas implicadas en la salud mental, un deterioro de los vínculos de confianza en sí mismos y en el otro correlativo a formas más o menos extremas de sufrimiento.

Un segundo criterio apunta, en otro nivel de análisis, a la estrecha relación entre las formas "psicopatológicas" del malestar y sus modalidades actuales de aparente resolución -específicamente, en el orden de las políticas de salud mental implementadas a través de planes y programas-. En este nivel de análisis, el vaciamiento de sentido subjetivo que se produce por las respuestas institucionales al problema de la salud mental en Chile no hace más que redoblar las fuentes mismas del malestar, toda vez que su medicalización privada somete al individuo a ser víctima de s mismo, alejado de los lazos de sociabilidad propios a un pacto social que se vuelve vínculo contractual de un bienestar improbable. 


\section{REFERENCIAS BIBLIOGRÁFICAS}

Almeida-Filho, N., "Becoming modern after all these years: social change and mental health in Latin America", en Culture, Medicine and Psychiatry, 22, 1998, págs. 285-316.

Araujo, K. y Martuccelli, D., Desafios comunes. Retrato de la sociedad chilena y sus individuos, Santiago: LOM, 2012.

Arteaga, C. y Martuccelli, D., "Neoliberalismo, corporativismo y experiencias posicionales. Los casos de Chile y Francia”, en Revista Mexicana de Sociología, 74, N², 2012, págs. 275-302.

Brunner, J.J., "Malestar en la sociedad chilena: ¿de qué, exactamente, estamos hablando?", en Estudios Públicos, № 72, Santiago de Chile: CEP, 1998, págs. 173-198.

Coleman, J., Foundations of Social Theory, Cambridge, Massachusetts: Harvard University Press, 1990.

De la Barra, F, Vicente, B., Rioseco, P., Saldivia, S. y Milpillán, R., "Prevalencia de trastornos psiquiátricos en poblaciones infanto-juveniles en Chile", 2012, [en prensa].

Duarte, D., Suicidio en Chile: un signo de exclusión, Santiago: Editorial Universitaria, 2007.

Freud, S., "El malestar en la cultura", en Obras Completas, Vol. XXI, Buenos Aires: Amorrortu, 2004, págs. 57-140.

Ehrenberg, A., La société du malaise, Paris: Odile Jacob, 2010.

Inglehart, R., Foa, R., Peterson, C. y Welzel, C., "Development, freedom and rising happiness. A global perspective (1981-2007)", en Perspectives on Psychological Science, Vol. 3, № 4, 2008, págs. 264-285.

Jirón, M., Machado, M. y Ruiz, I., "Consumo de antidepresivos en Chile entre 1992 y 2004", en Revista Médica de Chile, 136, 2008, págs. 1147-1154.

Kohn, R., Levav, I., Caldas de Almeida, J.M., Vicente, B., Andrade, L., Caraveo-Anduaga, J., Saxena, S., Saraceno, B., "Los trastornos mentales en América Latina y el Caribe: asunto prioritario para la salud pública", en Revista Panamericana de Salud Pública, 18 (4/5), 2005, págs. 229-240.

Layard, R., Happiness: Lessons from a New Science, New York: Penguin Press, 2005.

Lechner, N., "Desafíos de un desarrollo humano: individualización y capital social", en Obras escogidas, Vol. 2, Santiago: LOM, 2007.

Lipovetsky, G., Los tiempos hipermodernos, Barcelona: Anagrama, 2006.

Martuccelli, D., Cambio de rumbo. La sociedad a escala del individuo, Santiago: LOM, 2007.

Mayol, A. y Azócar, C., "Politización del malestar, movilización social y transformación ideológica: el caso 'Chile 2011", en Revista Polis, № 30, Santiago de Chile, 2011.

Ministerio de Salud [MINSAL], II Encuesta de Calidad de vida y Salud en Chile, Santiago: MINSAL, 2006, véase online en: http://epi.minsal.cl/epi/html/sdesalud/calidaddevida2006/Informe\%20Final\%20Encuesta\%20de\%20Calidad\%20de\%20Vida\%20y\%20Salud\%202006. pdf ]

- Estrategia nacional de salud mental: un salto adelante, Documento de trabajo, 2011, véase online en: http://www.minsal.gob.cl/portal/url/item/a73ea8bff9392ac4e0400101le011f16.pdf

_ Encuesta Nacional de Salud, Chile 2009-2010, Santiago: MINSAL-PUC, 2011b, véase online en: http://www.minsal.gob.cl/portal/url/item/bcb03d7bc28b64dfe040010165012d23.pdf

Miranda Hiriart, G., Alvarado, S., Kaufman, J., "Duración de las licencias médicas FONASA por trastornos mentales y del comportamiento", en Revista Médica de Chile, 140, 2012, 209-215.

Molina, R., George, M. et al., Estudio Nacional de Calidad de Vida relacionado con la Salud en Adolescentes Chilenos, Santiago: Proyecto Domeyko, Universidad de Chile, 2012. 
Moyano, E. y Barría, R., "Suicidio y producto interno bruto (PIB) en Chile: hacia un modelo predictivo", en Revista Latinoamericana de Psicología, Vol. 38, N², 2006, págs. 343-359.

OECD, Health Data 2011, 2011, véase online en: www.oecd.org/health

Organización Mundial de la Salud [OMS], Informe sobre la salud en el mundo. Salud mental: nuevos conocimientos, nuevas esperanzas, Ginebra: OMS, 2001, véase online en: http://www.who.int/ entity/whr/2001/en/whr01_es.pdf

_- Subsanar las desigualdades en una generación: alcanzar la equidad sanitaria actuando sobre los determinantes sociales de la salud, Informe final de la Comisión sobre Determinantes Sociales de la Salud, Ginebra: OMS, 2008.

PNUD, Desarrollo humano en Chile. Las paradojas de la modernización, Santiago: PNUD, 1998.

Rojas, I., "Plan Nacional de Prevención del Suicidio", en Jornada Suicidio en Chile. Perspectivas Actuales, Santiago de Chile, Pontificia Universidad Católica, 2008.

Ruiz, S., Reinoso, A., Asún, R., Aceituno, R., Ugarte, A., Jiménez, A., "Jóvenes secundarios de hoy: estudio de su visión de la sociedad desde los conceptos de anomia y alienación psicosocial", en Última Década, № 35, 2011, págs. 195-220.

Saffie, X., Pacientes (poli)consultantes a la atención primaria, Tesis (Magíster en Psicología Clínica), Universidad de Chile, Facultad de Ciencias Sociales, Santiago de Chile, 2011.

Veenhoven, R., "Quality of life in individualistic society: a comparison of 43 nations in the early 1990's”, en Social Indicators Research, 48, 1999, págs. 157-186.

Vicente, B., Rioseco, P., Saldivia, S., Kohn, R. y Torres, S. "Estudio chileno de prevalencia de patología psiquiátrica (DSM-III-R/CIDI)”, en Revista Médica de Chile, 130, 2002, págs. 527-536.

Vicente, B., Kohn, R., Saldivia, S. y Rioseco, P., "Carga del enfermar psíquico, barreras y brechas en la atención de salud mental en Chile", Revista Médica de Chile, 135, 2007, págs. 15911599. 